\title{
Estudo descritivo do tratamento do câncer em crianças e adolescentes no Sistema Único de Saúde do Brasil em 2014
}

\author{
Descriptive Study of the Treatment of Cancer in Children and Adolescents in the Brazilian \\ Unified Health System in 2014
Estudio Descriptivo del Tratamiento del Cáncer en Niños y Adolescentes en el Sistema Único de Salud de Brasil en 2014

Maria Beatriz Kneipp Dias ${ }^{1 *}$, Jeane Glaucia Tomazelli1, Denise Rangel Sant'Ana1, Antônio Carlos Antunes Bertholasce'.

\begin{abstract}
RESUMO
Objetivo: Analisar a produção de procedimentos quimioterápicos e radioterápicos registrados nos sistemas de informação referentes ao tratamento de crianças e adolescentes menores de 19 anos com câncer, considerando o tipo de habilitação oncológica dos estabelecimentos de saúde no Sistema Único de Saúde (SUS). Método: Estudo descritivo utilizando a base de dados do sistema de informação ambulatorial do SUS (SIA-SUS), a partir dos procedimentos de quimioterapia e de radioterapia registrados no ano de 2014, e análise das normativas existentes para tratamento oncológico pediátrico. Resultados: Dos 283 estabelecimentos habilitados em oncologia no SUS, 71 eram habilitados em oncologia pediátrica, entretanto 213 informaram realizar quimioterapia e 138 radioterapia em crianças e adolescentes. Os habilitados em oncologia pediátrica realizaram $75,1 \%$ do total de quimioterapia e $52,6 \%$ dos campos de radioterapia registrados. Houve variação na distribuição etária de quimioterapia segundo tipo de habilitação do estabelecimento. Serviços isolados de radioterapia informaram $34,4 \%$ dos campos irradiados em menores de um ano. Conclusão: Os procedimentos não foram realizados, em sua totalidade, em estabelecimentos preconizados conforme orientações existentes.

Palavras-chave: Neoplasias, Crianças, Adolescente, Terapêutica, Sistemas de Informação em Saúde.
\end{abstract}

\begin{abstract}
Objective: To analyze the production of chemotherapeutic and radiotherapeutic procedures registered in the information systems regarding the treatment of children and adolescents under 19 years of age with cancer, considering the type of oncological habilitation of health care establishments in the Unified Health System (SUS). Method: A descriptive study using the database of the SUS outpatient information system (SIA-SUS), based on the chemotherapy and radiotherapy procedures registered in the year 2014, and analysis of the existing norms for pediatric oncological treatment. Results: Of the 283 establishments qualified in oncology in the SUS, 71 were qualified in pediatric oncology, however 213 reported performing chemotherapy and 138 radiotherapy in children and adolescents. Pediatric oncology graduates performed $75.1 \%$ of the total chemotherapy and $52.6 \%$ of the registered radiotherapy fields. There was variation in the age distribution of chemotherapy according to the type of establishment habilitation. Isolated radiotherapy services reported $34.4 \%$ of the irradiated fields in children under one year. Conclusion: The procedures were not performed in their entirety in establishments recommended according to existing guidelines.
\end{abstract}

Key words: Neoplasms, Children, Adolescent, Therapeutics, Health Information Systems.

1 Instituto Nacional de Câncer José Alencar Gomes da Silva (INCA). Rio de Janeiro - RJ.

*E-mail: mdias@inca.gov.br

SUBMETIDO EM: 12/2018

ACEITO EM: 1/2019

PUBLICADO EM: $3 / 2019$

REAS/EJCH | Vol. 11 (6) | e522 | DOI: https://doi.org/10.25248/reas.e522.2019 Página 1 de 13 


\section{RESUMEN}

Objetivo: Analizar la producción de procedimientos quimioterápicos y radioterápicos en niños y adolescentes menores de 19 años con cáncer, considerando el tipo de habilitación oncológica de establecimientos del Sistema Único de Salud (SUS). Método: Estudio descriptivo utilizando una base de datos del sistema de información ambulatoria del SUS (SIA-SUS), a partir de los procedimientos de quimioterapia y radioterapia en el año 2014, y las normativas para el tratamento oncológico. Resultados: De los 283 establecimientos habilitados en oncología: 71 eran de oncología pediátrica, sin embargo 213 informaron realizar quimioterapia y 138 radioterapia. Los de oncología pediátrica realizaron el $75,1 \%$ del total de quimioterapia y el $52,6 \%$ de los campos de radioterapia. Hubo variación en la distribución por edades de la quimioterapia segundo tipo de habilitación. Los servicios aislados de radioterapia informaron el $34,4 \%$ de los campos irradiados en menores de un año. Conclusión: Los procedimientos no se realizaron, en totalidad, en establecimientos preconizados segun las orientaciones existentes.

Palabras-clave: Neoplasias, Niño, Adolescente, Terapéutica, Sistemas de Información en Salud.

\section{INTRODUÇÃO}

O câncer na criança e no adolescente é raro e sua incidência é maior nos países desenvolvidos sendo nestes, uma das principais causas de morte em crianças, ainda que $84 \%$ das crianças com câncer vivam nos países em desenvolvimento (MAGRATH et al., 2013).

Segundo estimativas do Globocan ocorreram no mundo cerca de 163.300 novos casos da doença e 80.000 mortes em crianças com até 14 anos de idade, em 2012 (FERLAY et al., 2013). A taxa de mortalidade entre países varia de acordo com o nível de desenvolvimento, sendo que, nos países mais desenvolvidos onde as taxas de incidência são maiores, as taxas de mortalidade são menores (MAGRATH et al., 2013; RODRIGUEZGALINDO et al., 2015).

Entre 1975 e 2010, a taxa de incidência de câncer pediátrico aumentou 0,6\% nos Estados Unidos da América (EUA) - comportamento também observado na Europa - enquanto a taxa de mortalidade reduziu em média 2,1\%, ao ano (WARD et al, 2014). Embora as causas do aumento sejam desconhecidas, devido a poucos fatores de risco para cânceres pediátricos serem conhecidos e, portanto, preveníveis (PAHO, 2014; WARD et al, 2014) é possível que este aumento esteja relacionado a fatores ambientais, melhorias do acesso e do diagnóstico e a introdução de tecnologias que melhoraram a acurácia diagnóstica (WARD et al, 2014). A redução na mortalidade, por sua vez, está associada aos avanços no tratamento (PAHO, 2014; PRITCHARD-JONES et al.,2013).

Para 2018, foram estimados no Brasil 12.500 casos novos de câncer em crianças e adolescentes até 19 anos, excluídos os tumores de pele não melanoma. Este quantitativo representa cerca de $3 \%$ do total de casos de câncer estimados para o período (INCA, 2017).

Por suas características o câncer em crianças e adolescentes é considerado altamente curável, principalmente, quando ocorre o diagnóstico e o tratamento em tempo oportuno (PAHO, 2014). Para que haja boa resposta aos tratamentos disponíveis é necessário que profissionais dos serviços de saúde da atenção básica identifiquem os casos suspeitos no estágio inicial da doença e os encaminhem para centros de referência com recursos diagnósticos e terapêuticos específicos para esta faixa etária (GRABOIS et al., 2011; MAGALHÃES et al., 2016; PAHO, 2014).

Para um tratamento mais efetivo é fundamental a disponibilidade de equipes especializadas com formação em pediatria e oncologia e de centros de tratamento bem estruturados com experiência no manejo clínico destes pacientes (AAP, 2004). O tratamento multidisciplinar (AAP, 2004; RODRIGUEZ-GALINDO et al., 2015) e em centros especializados (AAP, 2004; MAGALHÃES et al., 2016; PAHO, 2014) melhora a relação custo-eficácia, sendo necessário existir uma rede de investigadores experientes, composta por diversos profissionais de saúde (AAP, 2004; PRITCHARD-JONES et al.,2013; RODRIGUEZ-GALINDO et al., 2015). 
Nos últimos 25 anos, a utilização combinada de cirurgia, quimioterapia e radioterapia no tratamento do câncer infanto-juvenil vem aumentando, significativamente, a sobrevida a longo prazo (PAHO, 2014; PRITCHARDJONES et al.,2013). Essas modalidades terapêuticas são ofertadas no Sistema Único de Saúde (SUS), por meio de estabelecimentos habilitados em oncologia (BRASIL, 2014), com a orientação que os mesmos possuam Serviços de Oncologia Pediátrica.

A normativa governamental vigente - Portaria SAS/MS № 140 de 2014 - atualizou os critérios e parâmetros para organizar, planejar, monitorar, controlar e avaliar os estabelecimentos de saúde habilitados em oncologia, bem como, a definição das condições estruturais, de funcionamento e de recursos humanos para a habilitação destes estabelecimentos no âmbito do SUS (BRASIL, 2014). O documento define que, de modo geral, o tratamento oncológico deve ser realizado por hospitais gerais com capacidade para realizar a assistência oncológica. Quando isto acontece e existe a necessidade de promover acesso à confirmação diagnóstica e ao tratamento oncológico das pessoas com suspeita/confirmação de câncer, o estabelecimento de saúde é reconhecido pelo Ministério da Saúde e recebe habilitação como Centro de Assistência de Alta Complexidade em Oncologia (CACON) ou Unidade de Assistência de Alta Complexidade em Oncologia (UNACON), Entretanto, existem algumas patologias, faixa etária e tratamentos oncológicos que requerem infraestrutura - técnica, física, recursos humanos e equipamentos - específica. Por exemplo, para que o estabelecimento de saúde habilitado como CACON ou UNACON possa tratar crianças e adolescentes com câncer, ele precisa possuir Serviço de Oncologia Pediátrica conforme o desenho da Rede de Atenção à Saúde (RAS) estabelecido pela gestão do SUS (BRASIL, 2014).

Os estabelecimentos de saúde habilitados em oncologia e com capacidade instalada para tratar o câncer infanto-juvenil, conforme orientações governamentais respaldadas por especialistas na área, recebem diferentes tipos de habilitação do SUS. Eles podem ser habilitados como: (i) CACON com Serviço de Oncologia Pediátrica; (ii) UNACON com Serviço de Oncologia Pediátrica, o qual deve atender hematologia oncológica de crianças e adolescentes, facultando os cânceres raros; e (iii) UNACON exclusiva de Oncologia Pediátrica, a qual inclui os tumores sólidos e hematológicos de crianças e adolescentes (BRASIL, 2014).

O CACON, a UNACON com Serviço de Hematologia e a UNACON exclusiva de Hematologia podem atender crianças e adolescentes quando possuir médico especialista em hematologia pediátrica ou cancerologia pediátrica; quarto(s) exclusivo(s) com leito de isolamento e, sala de aplicação de quimioterápicos exclusiva para este grupo etário (BRASIL, 2014).

Quando o hospital for habilitado como CACON ou UNACON - ambos com Serviço de Oncologia Pediátrica ou UNACON exclusiva de Oncologia Pediátrica deve possuir, além dos requisitos gerais específicos para oncologia pediátrica, obrigatoriamente, os seguintes padrões estruturais: ambulatório de especialidades pediátricas; enfermarias com assistência exclusiva de internação pediátrica; serviço de cirurgia pediátrica com equipe de cirurgiões titulados em cirurgia pediátrica e, no mínimo, referências para cirurgia de cabeça e pescoço, cirurgia plástica, neurocirurgia, oftalmologia e ortopedia; centro cirúrgico com todos os atributos e equipamentos exigidos para a cirurgia pediátrica e, UTI pediátrica (BRASIL, 2014).

O número de hospitais habilitados como CACON e UNACON com Serviço de Oncologia Pediátrica ou com Serviço de Hematologia para tratar crianças e adolescentes, deve ser definido à luz dos parâmetros de planejamento e avaliação estabelecidos pela Portaria SAS/MS no 140 . Inclusive, para garantir a qualidade da assistência, o parâmetro mínimo de atendimento adotado é de, em média, 100 casos novos/ano, para cada área (pediatria e hematologia) (BRASIL, 2014).

Os procedimentos de quimioterapia (QT) e radioterapia (RT) realizados pelos hospitais habilitados devem ser informados no Sistema de Informação Ambulatorial (SIA/SUS), por meio da Autorização de Procedimentos de Alta Complexidade (APAC). Este quantitativo de produção, bem como, o número de casos de câncer tratados

REAS/EJCH | Vol. 11 (6) | e522 | DOI: https://doi.org/10.25248/reas.e522.2019 Página 3 de 13 
nos hospitais habilitados para tal, deve ser avaliado, monitorado e controlado pelos gestores do SUS, considerando os parâmetros existentes (BRASIL, 2016).

Diante do contexto apresentado, o objetivo deste estudo foi analisar a produção de procedimentos quimioterápicos e radioterápicos para tratar crianças e adolescentes menores de 19 anos com câncer, a partir do tipo de habilitação oncológica dos estabelecimentos, no âmbito do SUS.

\section{MÉTODOS}

Foi realizado um estudo descritivo a partir do quantitativo de procedimentos de QT e RT informados para tratamento oncológico de crianças e adolescentes com idade de 0 a 19 anos, registrados pelos estabelecimentos habilitados em oncologia, e, a atual norma administrativa que regulamenta o funcionamento destes serviços de saúde no SUS, a Portaria SAS/MS nำ140/ 2014.

Os dados de produção foram obtidos no Sistema de Informação Ambulatorial (SIA/SUS), disponibilizados no sítio do DATASUS (www.datasus.gov.br), referentes ao ano de 2014.

Foram contabilizados os procedimentos de QT e os números de campo de RT das seguintes doenças informadas: neoplasia maligna (C00 - C97), neoplasias in situ (D00 - D09) e as neoplasias de comportamento incerto ou desconhecido (D37 - D48), de acordo com a décima revisão da Classificação Estatística Internacional de Doenças e de Problemas de Saúde (CID-10) (OMS, 1996).

Os procedimentos de QT incluíram: as finalidades terapêuticas para crianças e adolescentes; os procedimentos especiais e as quimioterapias de adulto (adjuvante/profilática, controle temporário, curativa, paliativa e prévia). Essa última finalidade terapêutica foi incluída porque a regra de pagamento da QT para finalidade terapêutica de crianças e adolescentes é limitada a menores de 19 anos, e a tabela de procedimentos do SUS registra a idade máxima em até 22 anos com limite de idade do procedimento para menores de 19 anos. Sendo assim, o pagamento do procedimento de QT-para adolescentes de 19 anos é efetuado na finalidade adulto (BRASIL, 2015). Os procedimentos de hormonioterapia não foram incluídos, pois, geralmente, a terapia hormonal é usada para tratar cânceres de mama e de próstata, localizações que raramente ocorrem na população estudada (PRITCHARD-JONES et al.,2013).

A produção do número de campos realizados por acelerador linear e cobaltoterapia, ou mesmo por radioterapia estereotáxica, foram escolhidos por serem os procedimentos principais para o tratamento. Além disso, o número dos equipamentos de teleterapia profunda existentes nos serviços é que são utilizados como parâmetro para calcular a capacidade de tratamento de cada hospital habilitado como CACON e UNACON, segundo a portaria que regulamenta estas habilitações (BRASIL, 2014).

Para analisar onde o tratamento do câncer pediátrico foi realizado naquele ano, os estabelecimentos de saúde foram agrupados de acordo com suas habilitações, sendo elas:

- Com serviço de oncologia pediatria: CACON com Serviço de oncologia pediátrica; UNACON com Serviço de Oncologia Pediátrica; UNACON com Serviço de Radioterapia e de Oncologia Pediátrica; UNACON com Serviço de Hematologia e Oncologia Pediátrica; UNACON com Serviço de Radioterapia, Hematologia e Oncologia Pediátrica; UNACON exclusiva de Oncologia Pediátrica, UNACON exclusiva de Oncologia Pediátrica com Serviço de Radioterapia.

- Com hematologia: CACON; UNACON com Serviço de Hematologia; UNACON com Serviço de Radioterapia e Hematologia; UNACON exclusiva de Hematologia.

- Outras habilitações: UNACON; UNACON com Serviço de Radioterapia; também foram incluídos neste grupo os Serviços Isolados de Radioterapia autorizados pelo Ministério da Saúde.

REAS/EJCH | Vol. 11 (6) | e522 | DOI: https://doi.org/10.25248/reas.e522.2019 Página 4 de 13 
Os dados foram apresentados de acordo com a produção informada, faixa etária, tipo de habilitação e região do país. Foi utilizado o software R (R DEVELOPMENT CORE TEAM, 2013) na análise dos dados.

\section{RESULTADOS}

Em 2014, existiam no SUS 283 estabelecimentos de saúde habilitados em oncologia, dos quais 71 eram habilitados com Serviço de Oncologia Pediátrica (Tabela 1). Sendo que, o perfil de habilitação mais frequente desse último foi CACON com Serviço de Oncologia Pediátrica $(n=26)$, seguida de UNACON exclusiva de Oncologia Pediátrica $(n=13)$ e de UNACON com Serviço de Radioterapia, Hematologia e Oncologia Pediátrica $(n=12)$ (Tabela 1).

Tabela 1. Número de Estabelecimentos habilitados em oncologia, segundo tipo de habilitação. Brasil e grandes regiões, 2014.

\begin{tabular}{|c|c|c|c|c|c|c|c|}
\hline & Tipos de habilitações & Centro-Oeste & Nordeste & Norte & Sudeste & Sul & Brasil \\
\hline \multirow{7}{*}{ 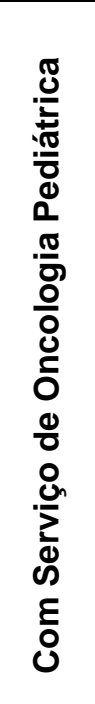 } & $\begin{array}{l}\text { CACON com serviço de oncologia } \\
\text { pediátrica* }\end{array}$ & 2 & 6 & 1 & 12 & 5 & 26 \\
\hline & $\begin{array}{l}\text { UNACON com serviço de oncologia } \\
\text { pediátrica }\end{array}$ & 1 & 1 & - & 5 & - & 7 \\
\hline & $\begin{array}{l}\text { UNACON com serviços de hematologia } \\
\text { e de oncologia pediátrica }\end{array}$ & - & 1 & - & 4 & 4 & 9 \\
\hline & $\begin{array}{l}\text { UNACON com serviços de radioterapia } \\
\text { e de oncologia pediátrica* }\end{array}$ & 1 & 1 & - & 1 & - & 3 \\
\hline & $\begin{array}{l}\text { UNACON com serviços de radioterapia, } \\
\text { de hematologia e de oncologia } \\
\text { pediátrica* }^{*}\end{array}$ & 1 & 1 & 1 & 6 & 3 & 12 \\
\hline & $\begin{array}{l}\text { UNACON exclusiva de oncologia } \\
\text { pediátrica }\end{array}$ & - & 4 & - & 6 & 3 & 13 \\
\hline & $\begin{array}{l}\text { UNACON exclusiva de oncologia } \\
\text { pediátrica com serviço de } \mathrm{RT}^{*}\end{array}$ & - & - & - & 1 & -- & 1 \\
\hline \multicolumn{2}{|r|}{$\begin{array}{c}\text { Total de habilitações com serviço de } \\
\text { oncologia pediátrica }\end{array}$} & 5 & 14 & 2 & 35 & 15 & 71 \\
\hline \multirow{4}{*}{ غ } & $\mathrm{CACON}^{*}$ & - & 4 & - & 10 & 4 & 18 \\
\hline & $\begin{array}{l}\text { UNACON com serviços de radioterapia } \\
\text { e de hematologia* }\end{array}$ & 1 & 3 & - & 18 & 10 & 32 \\
\hline & UNACON com serviço de hematologia & 2 & 4 & - & 11 & 4 & 21 \\
\hline & UNACON exclusiva de hematologia & - & 1 & - & 1 & - & 2 \\
\hline \multirow{4}{*}{$\stackrel{\substack{\pi \\
\frac{\pi}{3}}}{0}$} & UNACON com serviço de radioterapia* & 6 & 9 & 5 & 23 & 11 & 54 \\
\hline & UNACON & 6 & 19 & 3 & 30 & 20 & 78 \\
\hline & $\begin{array}{l}\text { Hospital geral com cirurgia de câncer de } \\
\text { complexo hospitalar }\end{array}$ & - & - & - & 7 & - & 7 \\
\hline & Total de Habilitações & 20 & 54 & 10 & 135 & 64 & 283 \\
\hline
\end{tabular}

Fonte: Portaria SAS/MS no140/ 2014. * Estabelecimentos habilitados com serviço de radioterapia 
A região Sudeste concentrou, aproximadamente, $50 \%(n=35)$ dos estabelecimentos com Serviço de Oncologia Pediátrica enquanto que, a região Norte apresentou apenas 2 estabelecimentos com essa habilitação.

Importante destacar que, aproximadamente, 60\% $(n=42)$ dos estabelecimentos com Serviço de Oncologia Pediátrica também eram habilitados com Serviço de Radioterapia (Tabela 1). Além disso, existiam 10 Serviços Isolados de Radioterapia autorizados a prestar atendimento radioterápico no SUS sendo, três na região Nordeste, cinco no Sudeste e dois no Sul (dados não apresentados).

Embora tenha sido constatado 71 estabelecimentos habilitados com Serviço de Oncologia Pediátrica, conforme os dados do SIA, pode-se observar que, 211 estabelecimentos apresentaram informação sobre produção de procedimentos de QT e 138 com informação de RT, independentemente de ser ou não habilitado para tratar crianças e adolescentes (Tabela 2).

Dentre os estabelecimentos habilitados com Serviço de Oncologia Pediátrica - onde se espera que todos produzam procedimentos de QT - dois não informaram produção e, daqueles habilitados com Serviço de Radioterapia, um deles não apresentou procedimentos de RT, todos localizados na região Sudeste (Tabela 2).

Dos estabelecimentos habilitados em oncologia, porém sem Serviço de Oncologia Pediátrica, quase 100\% dos CACON $(n=17)$ e, aproximadamente, $80 \%$ das UNACON com Serviços de Radioterapia e de Hematologia $(n=25)$ informaram procedimentos quimioterápicos e radioterápicos em menores de 19 anos. Em relação à $R T$, oito - dos dez serviços isolados - apresentaram produção (Tabela 2).

Foram informados 78.033 procedimentos de QT (Tabela 3) e 11.688 campos de RT (Tabela 4) em crianças e adolescentes em 2014. Os estabelecimentos habilitados como CACON com Serviço de Oncologia Pediátrica ( $n=26)$ foram os que mais informaram produção de QT $(n=25.972)$, seguidos dos 13 estabelecimentos habilitados como UNACON exclusiva de Oncologia Pediátrica $(n=15.162)$. Vale ressaltar que, dos 18.778 procedimentos quimioterápicos registrados em adolescentes com 15 a 19 anos, 30,6\% foram realizados em hospitais sem Serviço de Oncologia Pediátrica, ou seja, nos estabelecimentos agrupados com habilitação "com hematologia" e "outras" (Tabela 3).

A produção de procedimentos de QT em crianças e adolescentes foi de 1,2\% em menor de um ano, a 27,6\% na faixa etária de cinco a nove anos. Também houve variação no número de procedimentos entre as faixas etárias, segundo tipo da habilitação do estabelecimento, notadamente, naqueles habilitados como UNACON no grupo de habilitações "com hematologia", na qual a proporção na faixa etária de 15 a 19 anos variou de 40,1\% a $67,1 \%$ (Tabela 3).

Quanto à produção de procedimentos de RT, a menor proporção de número de campos realizados $(4,6 \%)$ também ocorreu em menores de um ano, porém variando de estabelecimentos que não tiveram nenhuma produção, até $34,4 \%$ do número de campos sendo realizados em Serviços Isolados de Radioterapia. A faixa etária com maior proporção de RT foi a de 15 a 19 anos (33,9\%), variando de 77,6\% nos estabelecimentos habilitados como UNACON com Serviços de Radioterapia e de Oncologia Pediátrica, a $20 \%$ no único estabelecimento habilitado como UNACON exclusiva de Oncologia Pediátrica com Serviço de Radioterapia. A diferença entre as faixas etárias foi significativa para todos estabelecimentos habilitados, exceto para o habilitado como UNACON exclusiva de Oncologia Pediátrica com Serviço de Radioterapia (Tabela 4). Dentre os estabelecimentos habilitados com Serviço de Oncologia Pediátrica, somente aqueles habilitados como CACON e UNACON com Serviços de Radioterapia e de Hematologia apresentaram produção de RT em menores de um ano. 
Tabela 2- Número de estabelecimentos habilitados em oncologia com informação de procedimentos de quimioterapia e radioterapia£ em crianças e adolescentes no SUS, por tipo de habilitação. Brasil e grandes regiões, 2014.

\begin{tabular}{|c|c|c|c|c|c|c|c|c|c|c|c|c|}
\hline \multirow{2}{*}{ Tipos de habilitações } & \multicolumn{2}{|c|}{ Centro-Oeste } & \multicolumn{2}{|c|}{ Nordeste } & \multicolumn{2}{|c|}{ Norte } & \multicolumn{2}{|c|}{ Sudeste } & \multicolumn{2}{|c|}{ Sul } & \multicolumn{2}{|c|}{ Brasil } \\
\hline & QT & $\mathrm{RT}$ & QT & $\mathrm{RT}$ & QT & $\mathrm{RT}$ & QT & RT & QT & $\mathrm{RT}$ & QT & RT \\
\hline $\begin{array}{l}\text { CACON com serviço de } \\
\text { oncologia pediátrica* }\end{array}$ & 2 & 2 & 6 & 6 & 1 & 1 & 12 & 12 & 5 & 5 & 26 & 26 \\
\hline $\begin{array}{l}\text { UNACON com serviço de } \\
\text { oncologia pediátrica }\end{array}$ & 1 & - & 1 & - & - & - & 4 & - & - & - & 6 & - \\
\hline $\begin{array}{l}\text { UNACON com serviços de } \\
\text { hematologia e de oncologia } \\
\text { pediátrica }\end{array}$ & - & - & 1 & - & - & - & 4 & - & 4 & - & 9 & - \\
\hline $\begin{array}{l}\text { UNACON com serviços de } \\
\text { radioterapia e de oncologia } \\
\text { pediátrica* }\end{array}$ & 1 & 1 & 1 & 1 & - & - & 1 & 1 & - & - & 3 & 3 \\
\hline $\begin{array}{l}\text { UNACON com serviços de } \\
\text { radioterapia, de hematologia } \\
\text { e de oncologia pediátrica* }\end{array}$ & 1 & 1 & 1 & 1 & 1 & 1 & 5 & 5 & 3 & 3 & 11 & 11 \\
\hline $\begin{array}{l}\text { UNACON exclusiva de } \\
\text { oncologia pediátrica }\end{array}$ & - & - & 4 & - & - & - & 6 & - & 3 & - & 13 & - \\
\hline $\begin{array}{l}\text { UNACON exclusiva de } \\
\text { oncologia pediátrica com } \\
\text { serviço de radioterapia* }\end{array}$ & - & - & - & - & - & - & 1 & 1 & - & - & 1 & 1 \\
\hline
\end{tabular}

$\mathrm{CACON}^{2}$

$-\quad-\quad-4$

44

17

UNACON com serviços de radioterapia e de hematologia*

$\begin{array}{llllllllllll}1 & 1 & 3 & 2 & - & - & 14 & 15 & 8 & 7 & 26 & 25\end{array}$

UNACON com serviço de hematologia $2-4 \quad 4 \quad-\quad-8 \quad-3 \quad 3 \quad-17$ -

UNACON exclusiva de hematologia

UNACON com serviço de radioterapia*

$\begin{array}{llllllllllll}4 & 5 & 7 & 9 & 4 & 6 & 14 & 18 & 8 & 9 & 37 & 47 \\ 5 & - & 8 & - & 3 & - & 13 & - & 13 & - & 42 & - \\ - & - & - & 2 & - & - & - & 4 & - & 2 & - & 8 \\ 17 & 10 & 41 & 25 & 9 & \mathbf{8} & 93 & 65 & 51 & 30 & 211 & 138\end{array}$

UNACON

mento acelerador linear (fótons ou fótons e elétrons), cobaltoterapia e radioterapia E número de campos por procedimento acelerador linear (fótons ou fóto
estereotáxica. * Estabelecimentos habilitados com serviço de radioterapia.

Serviços isolados de radioterapia Fonte: Sistema de Informação Ambulatorial. 
Tabela 3- Total de procedimentos de quimioterapia em crianças e adolescentes por faixa etária em estabelecimentos habilitados em Oncologia no SUS, Brasil, 2014.

\begin{tabular}{|c|c|c|c|c|c|c|c|c|c|c|c|c|}
\hline \multirow{2}{*}{ Habilitações } & \multicolumn{2}{|c|}{$<1$ ano } & \multicolumn{2}{|c|}{1 a 4 anos } & \multicolumn{2}{|c|}{5 a 9 anos } & \multicolumn{2}{|c|}{10 a 14 anos } & \multicolumn{2}{|c|}{15 a 19 anos } & \multirow{2}{*}{$\begin{array}{c}\text { Total } \\
\mathbf{n}\end{array}$} & \multirow{2}{*}{ p-valor } \\
\hline & $\mathbf{n}$ & $\%$ & $\mathbf{n}$ & $\%$ & $\mathbf{n}$ & $\%$ & $\mathbf{n}$ & $\%$ & $\mathbf{n}$ & $\%$ & & \\
\hline $\begin{array}{l}\text { CACON com Serviço } \\
\text { de Oncologia } \\
\text { Pediátrica }\end{array}$ & 388 & 1,5 & 6.453 & 24,8 & 6.687 & 25,7 & 5.896 & 22,7 & 6.548 & 25,2 & 25.972 & $<0,001$ \\
\hline $\begin{array}{l}\text { UNACON com Serviço } \\
\text { de Oncologia } \\
\text { Pediátrica }\end{array}$ & 17 & 1,3 & 329 & 24,5 & 344 & 25,6 & 382 & 28,4 & 272 & 20,2 & 1.344 & $<0,001$ \\
\hline $\begin{array}{l}\text { UNACON com } \\
\text { Serviços de } \\
\text { Hematologia e de } \\
\text { Oncologia Pediátrica }\end{array}$ & 92 & 1,1 & 1.764 & 20,5 & 2.458 & 28,6 & 2.115 & 24,6 & 2.168 & 25,2 & 8.597 & $<0,001$ \\
\hline $\begin{array}{l}\text { UNACON com } \\
\text { Serviços de } \\
\text { Radioterapia e de } \\
\text { Oncologia Pediátrica }\end{array}$ & 2 & 0,2 & 144 & 16,4 & 210 & 23,9 & 217 & 24,7 & 307 & 34,9 & 880 & $<0,001$ \\
\hline $\begin{array}{l}\text { UNACON com } \\
\text { Serviços de } \\
\text { Radioterapia, de } \\
\text { Hematologia e de } \\
\text { Oncologia Pediátrica }\end{array}$ & 57 & 1,2 & 1.112 & 24,3 & 1.303 & 28,5 & 1.041 & 22,8 & 1.060 & 23,2 & 4.573 & $<0,001$ \\
\hline $\begin{array}{l}\text { UNACON Exclusiva de } \\
\text { Oncologia Pediátrica }\end{array}$ & 240 & 1,6 & 4.244 & 28,0 & 4.540 & 29,9 & 3.900 & 25,7 & 2.238 & 14,8 & 15.162 & $<0,001$ \\
\hline $\begin{array}{l}\text { UNACON Exclusiva de } \\
\text { Oncologia Pediátrica } \\
\text { com Serviço de } \\
\text { Radioterapia }\end{array}$ & 36 & 1,7 & 478 & 22,9 & 564 & 27,0 & 573 & 27,4 & 439 & 21,0 & 2.090 & $<0,001$ \\
\hline$\overline{\mathrm{CACON}}$ & 57 & 0,8 & 1.913 & 26,0 & 2.125 & 28,9 & 1.562 & 21,3 & 1.692 & 23,0 & 7.349 & $<0,001$ \\
\hline $\begin{array}{l}\text { UNACON com } \\
\text { ServiçOs de } \\
\text { Radioterapia e de } \\
\text { Hematologia }\end{array}$ & 49 & 1,2 & 554 & 13,9 & 981 & 24,6 & 806 & 20,2 & 1.597 & 40,1 & 3.987 & $<0,001$ \\
\hline $\begin{array}{l}\text { UNACON Exclusiva de } \\
\text { Hematologia }\end{array}$ & - & & 74 & 11,5 & 159 & 24,8 & 146 & 22,8 & 262 & 40,9 & 641 & $<0,001$ \\
\hline $\begin{array}{l}\text { UNACON com Serviço } \\
\text { de Hematologia }\end{array}$ & - & & 61 & 8,7 & 95 & 13,6 & 74 & 10,6 & 469 & 67,1 & 699 & $<0,001$ \\
\hline $\begin{array}{l}\text { UNACON com Serviço } \\
\text { de Radioterapia }\end{array}$ & 28 & 0,6 & 1.137 & 23,7 & 1.519 & 31,7 & 1.066 & 22,2 & 1.047 & 21,8 & 4.797 & $<0,001$ \\
\hline UNACON & 7 & 0,4 & 367 & 18,9 & 557 & 28,7 & 332 & 17,1 & 679 & 35,0 & 1.942 & $<0,001$ \\
\hline
\end{tabular}

Fonte: Sistema de Informação Ambulatorial. Nota: Procedimentos com CID de neoplasia maligna e de comportamento incerto, conforme SIGTAP 
Tabela 4- Total de procedimentos por radioterapia em crianças e adolescentes por faixa etária em estabelecimentos habilitados em Oncologia no SUS, Brasil, 2014.

\begin{tabular}{|c|c|c|c|c|c|c|c|c|c|c|c|c|}
\hline \multirow{2}{*}{ Habilitações } & \multicolumn{2}{|c|}{$<1$ ano } & \multicolumn{2}{|c|}{1 a 4 anos } & \multicolumn{2}{|c|}{5 a 9 anos } & \multicolumn{2}{|c|}{10 a 14 anos } & \multicolumn{2}{|c|}{15 a 19 anos } & \multirow{2}{*}{$\begin{array}{c}\text { Total } \\
\mathbf{n}\end{array}$} & \multirow[t]{2}{*}{ p-valor } \\
\hline & $\mathbf{n}$ & $\%$ & $\mathbf{n}$ & $\%$ & $\mathbf{n}$ & $\%$ & $\mathbf{n}$ & $\%$ & $n$ & $\%$ & & \\
\hline $\begin{array}{l}\text { CACON com Serviço } \\
\text { de Oncologia } \\
\text { Pediátrica }\end{array}$ & 160 & 0,4 & 7.370 & 16,2 & 10.237 & 22,5 & 11.675 & 25,6 & 16.120 & 35,4 & 45.562 & $<0,001$ \\
\hline $\begin{array}{l}\text { UNACON com } \\
\text { Serviços de } \\
\text { Radioterapia e de } \\
\text { Oncologia Pediátrica }\end{array}$ & - & - & 34 & 4,7 & 23 & 3,2 & 105 & 14,5 & 561 & 77,6 & 723 & $<0,001$ \\
\hline $\begin{array}{l}\text { UNACON com } \\
\text { Serviços de } \\
\text { Radioterapia, de } \\
\text { Hematologia e de } \\
\text { Oncologia Pediátrica }\end{array}$ & 609 & 10,0 & 422 & 6,9 & 1.530 & 25,1 & 1.361 & 22,3 & 2.179 & 35,7 & 6.101 & $<0,001$ \\
\hline $\begin{array}{l}\text { UNACON Exclusiva } \\
\text { de Oncologia } \\
\text { Pediátrica com } \\
\text { Serviço de } \\
\text { Radioterapia }\end{array}$ & - & - & 1.668 & 26,4 & 1.305 & 20,6 & 2.093 & 33,1 & 1.263 & 20,0 & 6.329 & 0,215 \\
\hline CACON & 425 & 2,5 & 2.329 & 13,5 & 4.911 & 28,6 & 3.303 & 19,2 & 6.224 & 36,2 & 17.192 & $<0,001$ \\
\hline $\begin{array}{l}\text { Unacon com } \\
\text { Serviços de } \\
\text { Radioterapia e de } \\
\text { Hematologia }\end{array}$ & 549 & 5,5 & 666 & 6,7 & 1.944 & 19,6 & 3.033 & 30,6 & 3.711 & 37,5 & 9.903 & $<0,001$ \\
\hline $\begin{array}{l}\text { UNACON com } \\
\text { Serviço de } \\
\text { Radioterapia }\end{array}$ & 1.779 & 8,4 & 2.974 & 14,0 & 5.135 & 24,2 & 4.894 & 23,1 & 6.403 & 30,2 & 21.185 & $<0,001$ \\
\hline Isolada de RT & 1.614 & 34,4 & 20 & 0,4 & 685 & 14,6 & 926 & 19,7 & 1.448 & 30,9 & 4.693 & $<0,001$ \\
\hline Total Radioterapia & 5.136 & 4,6 & 15.483 & 13,9 & 25.770 & 23,1 & 27.390 & 24,5 & 37.909 & 33,9 & 111.688 & $<0,001$ \\
\hline
\end{tabular}

Fonte: Sistema de Informação Ambulatorial.

Enúmero de campos por procedimento acelerador linear (fótons ou fótons e elétrons), cobaltoterapia e radioterapia estereotáxica. Nota: Procedimentos com CID de neoplasia maligna e de comportamento incerto, conforme SIGTAP

Na análise dos grupos de habilitação, dos 78.033 procedimentos de QT em crianças e adolescentes, 75,1\% foram realizados em estabelecimentos com Serviço de Oncologia Pediátrica, 16,2\% com Serviço de Hematologia e $8,6 \%$ em Outros tipos de habilitações em oncologia. Os menores de um ano foram tratados, majoritariamente (85\%), em estabelecimentos com Serviço de Oncologia Pediátrica. Os estabelecimentos com Serviço de Hematologia foram responsáveis pelo atendimento de $21,4 \%$ dos adolescentes entre 15 e 19 anos enquanto que, 
os estabelecimentos com Outras Habilitações registraram menos de $10 \%$ dos procedimentos de QT em todas as faixas etárias, sendo 3,6\% em menores de um ano (Tabela 5).

Dos 111.688 campos de RT informados em crianças e adolescentes, 52,6\% foram realizados em estabelecimentos habilitados com Serviço de Oncologia Pediátrica, 24,3\% em estabelecimentos habilitados com Serviço de Hematologia e 23,2\% em Outras Habilitações, estando aqui incluídos $4 \%$ de procedimentos realizados por Serviços Isolados de Radioterapia autorizados. Em menores de um ano, 15\% dos campos de RT foram realizados em estabelecimentos com Serviço de Oncologia Pediátrica enquanto que, $66 \%$ dos campos foram registrados em estabelecimentos não habilitados em oncologia pediátrica. Nas demais faixas etárias não foram observadas variações importantes, mas os estratos foram estatisticamente diferentes ( $p$-valor $<0,0001)$ (Tabela 5).

Tabela 5 - Distribuição dos procedimentos de quimioterapia e radioterapia ${ }^{£}$ em crianças e adolescentes por faixa etária, segundo os grupos de habilitação e faixa etária. Brasil, 2014.

\begin{tabular}{|c|c|c|c|c|c|c|c|c|c|}
\hline \multirow{3}{*}{ 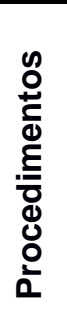 } & \multirow{3}{*}{ Faixa Etária } & \multicolumn{6}{|c|}{ Habilitações } & \multirow[t]{3}{*}{ Total } & \multirow[t]{3}{*}{ p-valor } \\
\hline & & \multicolumn{2}{|c|}{$\begin{array}{c}\text { Com Serviço de } \\
\text { Oncologia Pediátrica }\end{array}$} & \multicolumn{2}{|c|}{ Com Hematologia } & \multicolumn{2}{|c|}{ Outras } & & \\
\hline & & $\mathbf{n}$ & $\%$ & $\mathbf{n}$ & $\%$ & $\mathbf{n}$ & $\%$ & & \\
\hline \multirow{6}{*}{ 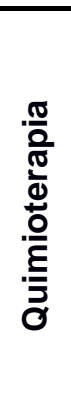 } & $<1$ ano & 832 & 85,5 & 106 & 10,9 & 35 & 3,6 & 973 & $<0,0001$ \\
\hline & 1 a 4 anos & 14.524 & 78 & 2.602 & 14 & 1.504 & 8,1 & 18.630 & $<0,0001$ \\
\hline & 5 a 9 anos & 16.106 & 74,8 & 3.360 & 15,6 & 2.076 & 9,6 & 21.542 & $<0,0001$ \\
\hline & 10 a 14 anos & 14.124 & 78 & 2.588 & 14,3 & 1.398 & 7,7 & 18.110 & $<0,0001$ \\
\hline & 15 a 19 anos & 13.032 & 69,4 & 4.020 & 21,4 & 1.726 & 9,2 & 18.778 & $<0,0001$ \\
\hline & Total & 58.618 & 75,1 & 12.676 & 16,2 & 6.739 & 8,6 & 78.033 & \\
\hline \multirow{6}{*}{ 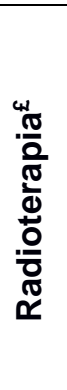 } & $<1$ ano & 769 & 15 & 974 & 19 & 3.393 & 66 & 5.136 & $<0,0001$ \\
\hline & 1 a 4 anos & 9.494 & 61,3 & 2.995 & 19,3 & 2.994 & 19,3 & 15.483 & $<0,0001$ \\
\hline & 5 a 9 anos & 13.095 & 50,8 & 6.855 & 26,6 & 5.820 & 22,6 & 25.770 & $<0,0001$ \\
\hline & 10 a 14 anos & 15.234 & 55,6 & 6.336 & 23,1 & 5.820 & 21,2 & 27.390 & $<0,0001$ \\
\hline & 15 a 19 anos & 20.123 & 53,1 & 9.935 & 26,2 & 7.851 & 20,7 & 37.909 & $<0,0001$ \\
\hline & Total & 58.715 & 52,6 & 27.095 & 24,3 & 25.878 & 23,2 & 111.688 & \\
\hline
\end{tabular}

Fonte: Sistema de Informação Ambulatorial.

Enúmero de campos por procedimento acelerador linear (fótons ou fótons e elétrons),cobaltoterapia e radioterapia estereotáxica. Nota: Procedimentos com CID de neoplasia maligna e de comportamento incerto, conforme SIGTAP 


\section{DISCUSSÃO}

A distribuição dos estabelecimentos de saúde com Serviço de Oncologia Pediátrica revela uma distribuição diferenciada, com menor número destes estabelecimentos na região Norte, onde também é encontrada a menor incidência de casos, 1.200 casos novos estimados para o ano de 2018 (INCA, 2017).

Os procedimentos de QT e RT informados por estabelecimentos de saúde habilitados em oncologia sem Serviço de Oncologia Pediátrica sinalizam a realização de tratamentos oncológicos fora dos centros de referências em oncologia pediátrica, como também observado no ano de 2013 (MAGALHÃES et al., 2016). Esta situação corrobora, de certa forma, uma análise realizada em 2016, com dados registrados no Registro Hospitalar de Câncer referentes no período 2009 a 2012, sobre o número de casos de câncer em crianças e adolescentes informados por estabelecimentos de saúde no SUS (INCA, 2016b). O referido levantamento mostrou que, dentre os 285 estabelecimentos habilitados em oncologia, 244 informaram casos de câncer em crianças e adolescentes. Destes, a maior parte $(n=216)$ registrou, no período, uma média de até 50 casos, sendo que, 174 estabelecimentos não eram habilitados com Serviço de Oncologia Pediátrica. Apenas nove estabelecimentos habilitados em pediatria oncológica possuíam média de casos igual a 100 ou mais (INCA, 2016b). Vale lembrar que, esse é o parâmetro mínimo que o estabelecimento habilitado deve atender por ano (BRASIL, 2014) e ele está correlacionado com o volume de atendimento e a expertise necessária para garantir a qualidade da assistência.

Uma revisão sistemática realizada em 2013, demonstrou melhores resultados de sobrevida e expertise profissional entre os hospitais especializados e com maior volume de atendimento em oncologia pediátrica (KNOPS et al.,2013). Portanto, este achado é extremamente relevante e evidencia a necessidade dos gestores do SUS, enquanto reguladores da Saúde no Brasil, avaliar, controlar, monitorar e auditar a produção de procedimentos oncológicos em pediatria, bem como, corrigir os rumos do processo de habilitação em oncologia dos estabelecimentos de saúde, a partir da definição das Regiões de Saúde e o planejamento da RAS para o controle do câncer, principalmente, com definição e implantação de protocolos de encaminhamento para o cuidado de crianças e adolescentes com suspeição/confirmação de câncer, abrangendo todos os níveis de atenção à saúde.

Um estudo sobre a assistência ao câncer infantil (GRABOIS et al., 2013) realizado entre os anos de 2000 a 2007, sugeriu que as crianças residentes nas regiões Norte e de regionais mais periféricas do Nordeste tiveram menor acesso à QT e RT quando comparados às residentes nas regiões Sudeste, Sul e Centro-Oeste. Atualmente, ao aplicar os parâmetros populacionais para habilitar estabelecimentos com Serviço de Oncologia Pediátrica (BRASIL, 2014), verifica-se a necessidade de 122 estabelecimentos desta natureza em todo o território nacional, devido ao provável déficit existente. Entretanto, se tal fato for justificar o atendimento oncológico infantojuvenil fora de estabelecimentos com Serviço de Oncologia Pediátrica, a qualidade do tratamento, bem como, os seus efeitos tardios (LOPES et al., 2000) devem ser avaliados e monitorados por órgãos públicos responsáveis pela autorização do pagamento da produção realizada em hospitais sem habilitação em oncologia pediátrica. E mais, o insuficiente número de casos atendidos nos estabelecimentos habilitados com Serviço de Oncologia Pediátrica sugere, fortemente, dificuldades no planejamento, organização e gestão tanto da RAS quanto na definição e pactuação das Regiões de Saúde.

Os estabelecimentos habilitados com Serviço de Oncologia Pediátrica foram os que mais apresentaram procedimentos de QT, um resultado esperado. Ainda assim, chama atenção três estabelecimentos habilitados como UNACON com Serviços de Radioterapia e de Oncologia Pediátrica cuja produção conjunta de QT foi de 880 procedimentos em todo ano de 2014. Embora hospitais habilitados em oncologia como "Outras" tenham realizado o menor percentual de procedimentos, não é esperado que estes apresentem produção de quimioterapia para tratar tumores hematopoiéticos e tumores de criança e adolescente.

REAS/EJCH | Vol. 11 (6) | e522 | DOI: https://doi.org/10.25248/reas.e522.2019 Página 11 de 13 
Apesar de 60\% dos estabelecimentos habilitados com Serviço de Oncologia Pediátrica possuir serviço de RT, estes foram responsáveis por, aproximadamente, $53 \%$ dos campos informados demonstrando que, alguns estabelecimentos, mesmo com Serviço de Oncologia Pediátrica, não têm realizado atendimento ou registrado sua produção na APAC. Isto foi observado na Região Sudeste, onde uma UNACON com Serviço de Radioterapia, de Hematologia e de Oncologia Pediátrica não apresentou produção de RT nem de QT em 2014.

A RT em menor de um ano em estabelecimentos com outras habilitações foi extremamente elevada, considerando os estabelecimentos habilitados com Serviço de Oncologia Pediátrica e os efeitos prejudiciais da radioterapia em crianças (KRASIN et al.,2010; NCCN, 2014). Uma possível hipótese para este resultado é o erro no registro da idade, visto ter sido identificado que, entre as principais neoplasias em crianças e adolescentes informadas por estabelecimentos sem habilitação em oncologia estão o câncer de mama e de próstata (INCA, 2016b).

Por fim, existem várias questões a serem enfrentadas pelos gestores do SUS juntos com os profissionais especializados em oncologia pediátrica. Algumas delas passam pela definição e divulgação de fluxos de encaminhamento para hospitais de referência para o tratamento do câncer infantil, assegurando a confirmação diagnóstica em tempo oportuno e tratamento de qualidade; por maior controle do Estado brasileiro no custeio dos procedimentos oncológicos em crianças e adolescentes realizados em estabelecimentos de saúde sem habilitação em oncologia pediátrica (MAGALHÃES et al., 2016). Aliado a isso, é importante uma proatividade na articulação e estruturação de estabelecimentos de saúde suficientes e com capacidade de tratar crianças e adolescentes com câncer e cumprir os padrões exigidos nas regulamentações vigentes (BRASIL, 2014), elaboradas a partir das melhores evidências científicas.

Ainda vale destacar que este estudo avança no conhecimento ao descrever a oferta de tratamento quimio e radioterápico no SUS pelo tipo de habilitação dos estabelecimentos em oncologia. Este diferencial permitiu apontar (i) diferenças importantes quanto o registro de tratamento radioterápico em menores de 1 ano e (ii) a necessidade estudos mais específicos que avaliem o impacto do tratamento oncológico em crianças e adolescentes segundo a assistência prestada pelos diferentes tipos de habilitações.

\section{CONCLUSÃO}

Através da análise da produção de procedimentos de quimioterapia e radioterapia em crianças e adolescentes menores de 19 anos com câncer foi possível verificar que a oferta do tratamento oncológico no SUS não se limita às unidades/centros habilitados em oncologia pediátrica, sendo oferecido em diferentes estabelecimentos habilitados em oncologia, mesmo em regiões que dispõe de estrutura disponível para atendimento. Esta situação vai contra as recomendações internacionais e a regulamentação nacional que aponta a necessidade de garantia de um atendimento em centros especializados que permitam melhor diagnóstico, tratamento e expertise na atenção integral à criança e adolescente com câncer.

\section{REFERÊNCIAS}

1. AMERICAN ACADEMY OF PEDIATRICS. Guidelines for the Pediatric Cancer Center and Role of Such Centers in Diagnosis and Treatment. Section on Hematology/Oncology. PEDIATRICS, v. 99, n. 1, p. 139-141, 1 jan. 1997.

2. BRASIL. MINISTÉRIO DA SAÚDE. SECRETARIA DE ATENÇÃO À SAÚDE. Portaria N¹40. Redefine os critérios e parâmetros para organização, planejamento, monitoramento, controle e avaliação dos estabelecimentos de saúde habilitados na atenção especializada em oncologia e define as condições estruturais, de funcionamento e de recursos humanos para a habilitação destes estabelecimentos no âmbito do Sistema Único de Saúde (SUS). 27 fev. 2014.

3. BRASIL. MINISTÉRIO DA SAÚDE. SECRETARIA DE ATENÇÃO À SAÚDE. DEPARTAMENTO DE REGULAÇÃO, AVALIAÇÃO E CONTROLE. COORDENAÇÃO GERAL DE SISTEMAS DE INFORMAÇÃO. SIA/SUS Sistema de

REAS/EJCH | Vol. 11 (6) | e522 | DOI: https://doi.org/10.25248/reas.e522.2019 Página 12 de 13 
Informações Ambulatoriais. Oncologia. Manual de Bases Técnicas. 23ā. ed. Brasília, DF: Ministério da Saúde, 2016, $144 \mathrm{p}$.

4. FERLAY, J. et al. GLOBOCAN 2012 v1.0, Cancer Incidence and Mortality Worldwide: IARC CancerBase No. 11 Disponível em: <http://globocan.iarc.fr, acessado em 15/05/2017. Lyon, France: International Agency for Research on Cancer, 2013.

5. GRABOIS, M. F. et al. Assistência ao câncer entre criancas e adolescentes: mapeamento dos fluxos origem-destino no Brasil. Revista de Saúde Pública, v. 47, n. 2, p. 368-378, jun. 2013.

6. GRABOIS, M. F. et al. Childhood cancer and pediatric oncologic care in Brazil: access and equity. Cadernos de Saúde Pública, v. 27, n. 9, p. 1711-1720, set. 2011.

7. INSTITUTO NACIONAL DE CÂNCER JOSÉ ALENCAR GOMES DA SILVA. COORDENAÇÃO DE PREVENÇÃO E VIGILÂNCIA. Estimativa 2018: incidência de câncer no Brasil. Rio de Janeiro: INCA, 2017.

8. INSTITUTO NACIONAL DE CÂNCER JOSÉ DE ALENCAR GOMES DA SILVA. Informativo Detecção Precoce. Monitoramento das ações de controle dos cânceres em crianças e adolescentes. Ano 4 , n 2 INCA, 2016.

9. KNOPS, R. R. G. et al. The volume effect in paediatric oncology: a systematic review. Annals of Oncology, v. 24, n. 7, p. 1749-1753, jul. 2013.

10. KRASIN, M. J. et al. Radiation-Related Treatment Effects Across the Age Spectrum: Differences and Similarities or What the Old and Young Can Learn from Each Other. Seminars in Radiation Oncology, v. 20, n. 1, p. 21-29, jan. 2010.

11. LOPES, L. F. et al. Os efeitos tardios do tratamento do câncer infantil. Revista da Associação Médica Brasileira, v. 46 , n. 3, p. 277-284, set. 2000.

12. MAGAlHÃES, I. Q. et al. Oncologia Pediátrica no Brasil: Por que há Poucos Avanços? Revista Brasileira de Cancerologia. Revista Brasileira de Cancerologia, v. 62, n. 4, p. 337-341, 2016.

13. MAGRATH, I. et al. Paediatric cancer in low-income and middle-income countries. The Lancet Oncology, v. 14, n. 3, p. e104-e116, mar. 2013.

14. NATIONAL COMPREHENSIVE CANCER NETWORK. Survivorship: Cognitive Function, Version 1.2014. Journal of the National Comprehensive Cancer Network, v. 12, n. 7, p. 976-985, 2014.

15. ORGANIZAÇÃO MUNDIAL DA SAÚDE. Cid-10: classificação estatística internacional de doenças e problemas relacionados à saúde. São Paulo: Edusp, 1996.

16. PAN AMERICAN HEALTH ORGANIZATION. Early Diagnosis of Childhood Cancer. Washington,: PAHO, 2014.

17. PRITCHARD-JONES, K. et al. Sustaining innovation and improvement in the treatment of childhood cancer: lessons from high-income countries. The Lancet Oncology, v. 14, n. 3, p. e95-e103, mar. 2013.

18. R DEVELOPMENT CORE TEAM. R: A language and environment for statistical computing. Vienna, Austria: $R$ Foundation for Statistical Computing, 2013.

19. RODRIGUEZ-GALINDO, C. et al. Toward the Cure of All Children With Cancer Through Collaborative Efforts: Pediatric Oncology As a Global Challenge. Journal of Clinical Oncology, v. 33, n. 27, p. 3065-3073, 20 set. 2015.

20. WARD, E. et al. Childhood and adolescent cancer statistics, 2014: Cancer in Children and Adolescents. CA: A Cancer Journal for Clinicians, v. 64, n. 2, p. 83-103, mar. 2014. 